Original Paper http://ajol.info/index.php/ijbcs http://indexmedicus.afro.who.int

\title{
Qualité physico-chimique et bactériologique des eaux souterraines et risques sanitaires dans quelques quartiers de Yaoundé VII, Cameroun
}

\author{
Diane Armelle MOUSSIMA YAKA ${ }^{1,3 *}$, Ange Alex TIEMENI ${ }^{2}$, Bertrand ZING ZING ${ }^{1}$, \\ Thérèse Line Laure JOKAM NENKAM ${ }^{3}$, Amina ABOUBAKAR ${ }^{1}$, \\ Aline Beatrice NZEKET ${ }^{1}$, Brice Hermann FOKOUONG TCHOLONG ${ }^{1}$ et \\ Yvette Clarisse MFOPOU MEWOUO ${ }^{1}$
}

\author{
${ }^{1}$ Laboratoire d'Analyses des Sols, Plantes, Eaux et Engrais, Coordination Scientifique Forêt, Sol et \\ Environnement, Institut de Recherche Agricole pour le Développement, Yaoundé, Cameroun. B.P. : 2123 \\ Yaoundé, Cameroun. \\ ${ }^{2}$ Laboratoire d'Analyses Microbiologique et Toxicologique, Département de Biochimie, Faculté des Sciences, \\ Université de Douala, Cameroun. B.P. :24157 Douala, Cameroun. \\ ${ }^{3}$ Laboratoire d'Hydrobiologie et Environnement, Département de Biologie des Organismes \\ Animaux, Faculté des Sciences, Université de Yaoundé 1, Cameroun. B.P. : 812 Yaoundé, Cameroun. \\ *Auteur correspondant ; E-mail: dmoussimav@gmail.com, Tél : +237675 777441
}

\section{RÉSUME}

$\mathrm{Au}$ Cameroun, l'accès aux systèmes d'alimentation en eau potable en milieu urbain apparaît sélectif. Les populations des quartiers situés en périphérie ont recours aux puits et sources pour assouvir leurs besoins. La présente étude a pour objectif d'évaluer la qualité physico-chimique et bactériologique des eaux souterraines couramment utilisées dans quelques quartiers de l'arrondissement de Yaoundé VII. 24 échantillons d'eaux prélevés dans 6 sources $\left(\mathrm{S}_{1}\right.$ à $\left.\mathrm{S}_{6}\right)$ et 2 puits $\left(\mathrm{W}_{1}\right.$ et $\left.\mathrm{W}_{2}\right)$ ont été analysés suivant des méthodes normalisées. Les températures relevées, proches de celle de l'air, révèlent le caractère libre des nappes étudiées. Celles-ci subiraient l'influence directe des eaux d'infiltration. Les stations $\mathrm{S}_{1}, \mathrm{~W}_{1}$ et $\mathrm{W}_{2}$ présentent des valeurs en nitrates supérieures à $50 \mathrm{mg} / \mathrm{l}$ (norme OMS). La consommation de leurs eaux expose la population jeune à la méthémoglobinémie. Contrairement aux recommandations de la norme camerounaise, les indicateurs de pollution fécale et les bactéries du genre Salmonella, ont été isolées dans les eaux analysées. Une analyse en composante principale ainsi qu'un dendrogramme ont permis de classer les points d'échantillonnage, selon l'ordre croissant de pollution organique suivant : $\left\{\mathrm{S}_{6}\right\},\left(\mathrm{S}_{3}, \mathrm{~S}_{4}, \mathrm{~S}_{5}\right),\left(\mathrm{S}_{1}, \mathrm{~S}_{2}\right),\left\{\mathrm{W}_{1}\right\},\left\{\mathrm{W}_{2}\right\}$. Cependant, du point de vue pathogénique, $\mathrm{S}_{6}, \mathrm{~S}_{3}, \mathrm{~S}_{4}, \mathrm{~S}_{5}$ sont les plus à craindre.

(C) 2020 International Formulae Group. All rights reserved.

Mots clés : Qualité des eaux, puits, sources, Yaoundé, risques sanitaires.

\section{Physico-chemical and bacteriological quality of groundwater and health risks in some districts of Yaoundé VII, Cameroon}

\begin{abstract}
In Cameroon, access to drinking water supply systems in urban areas appears to be selective. The populations located in the peripheral zone use wells and springs to satisfy their needs. This study aims to assess the physico-chemical and bacteriological quality of the groundwater commonly used in some districts of
\end{abstract}


Yaoundé VII. 24 water samples taken from 6 sources $\left(S_{1}\right.$ to $\left.S_{6}\right)$ and 2 wells $\left(W_{1}\right.$ and $\left.W_{2}\right)$ were analyzed according to standardized methods. The temperatures recorded, close to that of the air, reveal the free nature of the aquifers studied which are directly influenced by the infiltration waters. Samples points $\mathrm{S}_{1}, \mathrm{~W}_{1}$ and $\mathrm{W}_{2}$ presented concentration of nitrate above $50 \mathrm{mg} / \mathrm{l}$ (WHO standard). The consumption of their waters exposes young people to methemoglobinemia. On contrary to the recommendations of the cameroonian standard, the indicators of faecal pollution and bacteria of the genus Salmonella, were isolated in the waters analyzed. A principal component analysis as well as a dendrogram permit to classify the sampling points, according to the following increasing order of organic pollution: $\left\{\mathrm{S}_{6}\right\},\left(\mathrm{S}_{3}, \mathrm{~S}_{4}, \mathrm{~S}_{5}\right),\left(\mathrm{S}_{1}, \mathrm{~S}_{2}\right),\left\{\mathrm{W}_{1}\right\},\left\{\mathrm{W}_{2}\right\}$. However, from a pathogenic point of view, $\mathrm{S}_{6}, \mathrm{~S}_{3}, \mathrm{~S}_{4}, \mathrm{~S}_{5}$, are the most to be feared.

(C) 2020 International Formulae Group. All rights reserved.

Keywords: Water quality, Dug wells, spring, Yaoundé, sanitary risks.

\section{INTRODUCTION}

L'accès à l'eau potable est un facteur déterminant pour le développement aussi bien socio-économique, qu'environnemental d'une population. Cette problématique hautement préoccupante pour la communauté internationale, fait l'objet du sixième objectif de développement durable (UNRIC, 2020). Une eau de consommation devrait respecter les directives de qualité de l'eau de boisson fixées par l'OMS. Ce sont des exigences raisonnables minimales s'appliquant aux pratiques sans risque, destinées à protéger la santé des consommateurs (OMS, 2004). De ces directives, naissent des normes nationales qui intègrent le contexte environnemental, social, économique et culturel de chaque pays. La surveillance de la qualité de l'eau suivant ces normes permet d'assurer son intégrité et par là même, la santé des consommateurs.

$\mathrm{Au}$ Cameroun, les grandes villes qui abritent la majorité de la population sont à $86,2 \%$ équipées en systèmes d'alimentation en eau potable (DSCE, 2009). Cependant, à peine $25 \%$ des ménages y ont réellement accès de manière continue (Rapport National du Cameroun pour HABITAT-III, 2015). A l'issue du Conseil de cabinet qui s'est tenu le 31 mai 2018, le Ministre de l'Eau et de l'Energie a procédé au lancement de plusieurs projets de renforcement du système de production d'eau potable en milieu urbain. Il s'agissait, entre autres, du projet d'alimentation en eau potable de la ville de Yaoundé et ses environs, destiné à accroître la production de $300000 \mathrm{~m}^{3}$ par jour ; du projet de construction de 11 forages dans la ville de
Douala permettant d'injecter $30000 \mathrm{~m}^{3}$ d'eau dans le réseau de distribution ou encore des projets d'alimentation en eau potable des villes de Bertoua, Ngaoundéré ainsi qu'Edéa.

Toutefois, malgré ces efforts, l'accès à l'eau potable en milieu urbain apparaît toujours sélectif et, les populations des quartiers situés en périphérie n'y ont que difficilement accès. Dans ces zones, l'eau ne provenant que rarement du réseau public d'approvisionnement, d'autres sources telles que les puits et sources sont fréquemment utilisées pour les travaux domestiques, la cuisson des aliments, l'hygiène corporelle et même la consommation directe. Ceci n'est pas sans risque pour la santé des citadins. En effet, la conformité de ces eaux aux exigences de la norme camerounaise pourrait être mise en cause.

Dans la ville de Yaoundé, 3\% des ménages ne disposent pas de toilettes et défèquent à l'air libre (Nzouebet, 2019). Envirron 52\% des ménages canalisent les eaux des toilettes vers les fosses septiques, $34 \%$ déversent ces eaux dans un trou, et jusqu' à $15 \%$ sollicitent les rigoles qui desservent les quartiers pour évacuer leurs eaux usées des toilettes (INS-BGR, 2013). Du fait de leur mauvaise gestion, ces déchets pourraient constituer une source importante de contamination de la nappe phréatique.

C'est ainsi que nous avons entrepris de mener la présente étude dont l'objectif était d'évaluer la qualité bactériologique et physico-chimique des eaux d'usage courante, d'origine souterraine dans quelques quartiers secondaires de la ville de Yaoundé. 


\section{MATÉRIEL ET MÉTHODES \\ Site d'étude}

La commune d'arrondissement de Yaoundé VII est située dans la région du centre entre $3^{\circ} 50$ et $3^{\circ} 55$ de latitude Nord et entre $11^{\circ} 25$ et $11^{\circ} 29$ de longitude Est. Créée en 2007, elle est la dernière-née des communes de Yaoundé (Décret $\mathrm{N}^{\circ}$ 2007/115 du 23 avril 2007). Elle s'étend sur une superficie de $34.9 \mathrm{~km}^{2}$. En 2014, elle abritait déjà une population de 366666 habitants (Banque Mondiale-MINDHU, 2017). Cette commune d'arrondissement couvre 17 quartiers tous sujets au problème d'enclavement. Parmi ces quartiers, trois des plus densément peuplés ont été retenus pour la présente étude. Il s'agit d'OyomAbang, Nkolbisson et AkokNdoé. Dans ces trois localités, l'activité économique se résume aux petits commerces, l'artisanat et l'agriculture. Elles sont très peu desservies par la société d'adduction en eau potable du Cameroun (CAMWATER). Pourtant, caractérisées par des constructions anarchiques et une gestion précaire des déchets, ces localités font régulièrement l'objet des inondations susceptibles de menacer l'intégrité de la nappe phréatique. La Figure 1 illustre la représentation spatiale des différents points d'échantillonnage retenus sur le site d'étude.

\section{Échantillonnage}

Après prospection des sources et puits des quartiers OyomAbang, Nkolbisson et Akok-Ndoé, 24 points d'eau souterraine ont été recensés. Huit (8) ont été retenus sur la base d'un échantillonnage aléatoire. Soit 6 sources et 2 puits dont la description et la localisation sont consignées dans le Tableau 1. Les coordonnées GPS ont été prises sur le terrain à l'aide d'un récepteur de marque GARMIN.

Les prélèvements ont été faits durant la petite saison sèche (mi-juin à mi-août 2018), à une fréquence mensuelle. L'échantillonnage des eaux souterraines s'est fait suivant la méthode de Rodier et al. (2009). Pour les analyses physico-chimiques, l'eau a été prélevé dans des bouteilles en polyéthylènes à double bouchage de $1000 \mathrm{cc}$ préalablement étiquetées, lavées et rincées. Pour ce qui est des échantillons d'eau destinés aux analyses bactériologiques, des bidons de 51 préalablement stérilisées à l'autoclave et étiquetés ont été utilisés. Le $\mathrm{pH}$, la température, la conductivité électrique de l'eau ainsi que les TDS ont été mesurés in situ à l'aide d'un multi paramètre de marque Thermo-scientific ORION STAR 225. Les échantillons d'eau ont ensuite été transportés en enceinte réfrigérée jusqu'au Campus de l'Institut de Recherche Agricole pour le Développement (IRAD) pour les examens complémentaires. Des analyses physicochimiques ont été faites au Laboratoire d'Analyses des Sols, Plantes, Eaux et Engrais (LASPEE) de l'IRAD, tandis que les analyses bactériologiques ont été effectuées au Laboratoire de Phytopathologie de la même institution.

\section{Analyse des paramètres physico-chimiques}

Les paramètres analysés ainsi que les techniques utilisées sont présentés dans le Tableau 2. La dureté totale exprimée en degré français $\left({ }^{\circ} \mathrm{f}\right)$ a été calculée par la formule : Dureté totale $=$ titre hydrotimétrie $(\mathrm{TH})=$ $\frac{\left[\mathrm{Ca}^{2}\right]}{4}+\frac{\left[\mathrm{Mg}^{2}\right]}{2,4}$

Remarque : $1^{\circ} \mathrm{f}=4 \mathrm{mg} / \mathrm{L}$ de calcium $=2,4$ $\mathrm{mg} / \mathrm{L}$ de magnésium.

$\left[\mathrm{Ca}^{2}\right]_{=}$concentration en calcium $(\mathrm{mg} / \mathrm{L})$ dans l'échantillon d'eau.

$\left[\mathrm{Mg}^{2}\right]=$ concentration en magnésium (mg/L) dans l'échantillon d'eau.

\section{Analyse des paramètres biologiques}

La flore aérobie mésophile totale (FAMT), des indicateurs de pollution fécale, notamment les entérocoques intestinaux et les streptocoques fécaux ainsi qu'un germe pathogène d'un très grand intérêt sanitaire au Cameroun à savoir Salmonella spp ont été recherchés dans ces eaux. La NC 207 (2014) exigeant une absence totale des indicateurs de pollution fécale dans $100 \mathrm{ml}$ d'eau ainsi que du germe pathogène recherché dans $5 \mathrm{~L}$ d'eau de consommation, cette étude s'est limitée à rechercher leur présence dans le milieu sans toutefois les quantifier. 


\section{Flore Aérobie Mésophile Totale}

Le dénombrement de la flore aérobie mésophile totale a été fait suivant la NF EN ISO 6222 (1999). Un volume d'1 ml d'échantillon d'eau a été ensemencé en profondeur dans la gélose PCA (la gélose glucosée à l'extrait de levure appelée par les Anglo-Saxons "Plate Count Agar"). Les boîtes de Pétri contenant la préparation ont ensuite été incubées à l'étuve à une température de 37 ${ }^{\circ} \mathrm{C}$ pendant $48 \mathrm{~h}$. Le résultat a été exprimé en Unité Formant Colonie (UFC) par millilitre (ml) d'échantillon d'eau.

\section{Indicateurs de pollution fécale}

L'identification des entérocoques intestinaux et des streptocoques fécaux dans l'eau a été faite sur la base Présence/Absence dans $1600 \mathrm{ml}(1,61)$ en utilisant les milieux de culture (Slanetz Bartley ; BEA) et les paramètres d'incubation recommandés par la NF EN ISO 7899-2 (2000).

Un volume de $5 \mathrm{ml}$ du culot de l'échantillon d'eau préalablement concentré, a été pré- enrichie à l'eau peptonnée tamponnée (EPT) puis incubés pendant $18 \mathrm{~h}$ à $37^{\circ} \mathrm{C}$. A suivie l'ensemencement du bouillon EPT par strie $\mathrm{Z}$ sur gélose Slanetz et Bartley et incubation à $37^{\circ} \mathrm{C}$ pendant $48 \mathrm{~h}$. Les colonies rouges bombées observées sur le milieu ont été repiquées et incubées à nouveau à $44^{\circ} \mathrm{C}$ pendant $24 \mathrm{~h}$ sur gélose BEA pour confirmation. Enfin, la coloration de Gram et le test biochimique à la catalase ont été effectués à partir des colonies noires obtenues sur gélose BEA (Bile Esculine Azide de sodium) pour distinguer les entérocoques intestinaux des streptocoques fécaux.

\section{Salmonella spp.}

La recherche des salmonelles dans l'eau a été faite en quatre étapes selon les recommandations de la NF ISO 19250 (NF, 2010). Il s'est agit du pré-enrichissement de l'échantillon d'eau à l'EPT pour régénérer les bactéries présentes dans l'eau ; de l'enrichissement sélectif en utilisant le bouillon sélectif de Sélénite afin de permettre le développement de Salmonella au détriment des autres bactéries concurrentes $(2 \mathrm{ml}$ d'EPT pré-enrichie est introduite dans $20 \mathrm{ml}$ de bouillon sélénite et incubée à $37^{\circ} \mathrm{C}$ pendant 24 heures); de l'isolement par ensemencement en stries $\mathrm{Z}$ du bouillon sélénite sur le milieu spécifique de gélose Hektoen qui a ensuite été incubé à $37{ }^{\circ} \mathrm{C}$ pendant 24 heures. Enfin l'étape confirmation par des tests biochimiques à partir des Salmonella spp présomptives (colonies vertes ou bleues sans centre noir et vertes avec centre noir) isolées sur gélose Hektoen. Les tests biochimiques en tube sur milieu Kligler Hajna, le test de catalase, la coloration de Gram ont été effectués. Ces colonies ont également été observées à l'état frais dans le but de confirmer la mobilité type péritriche de Salmonella spp.

\section{Contrôle qualité}

Chaque échantillon d'eau prélevé a été analysé en 3 répétitions afin de limiter les erreurs de manipulation. Toujours dans le but de contrôler les dérives, un échantillon témoin (échantillon dont la valeur réelle est connue) a été inséré dans chaque série d'analyse. Ces mesures ont été appliquées aussi bien pour la caractérisation physico-chimique que la caractérisation bactériologique des eaux.

\section{Analyses statistiques et réalisation des cartes}

Les analyses statistiques ont été effectuées à l'aide des logiciels libres $\mathrm{R}$ version 3.2.3 et DIAGRAMME de Roland Smiler. Le logiciel $\mathrm{R}$ a permis : d'établir la statistique descriptive des sites d'étude (minimum, médiane, moyenne, maximum, analyse en composante principale); d'évaluer la robustesse des relations entre les paramètres physico-chimiques et biologiques (matrice de corrélation); de rechercher les affinités entre les stations d'échantillonnage (dendrogramme). Le logiciel DIAGRAMME développé par Roland Smiller du département d'Hydrogéologie de l'université d'Avignon en France, a quant à lui, permis de définir le faciès chimique des eaux (diagramme de Piper). Les cartes ont été réalisées à l'aide des logiciels QGIS 2.18.17 et GIMP 2.8. 


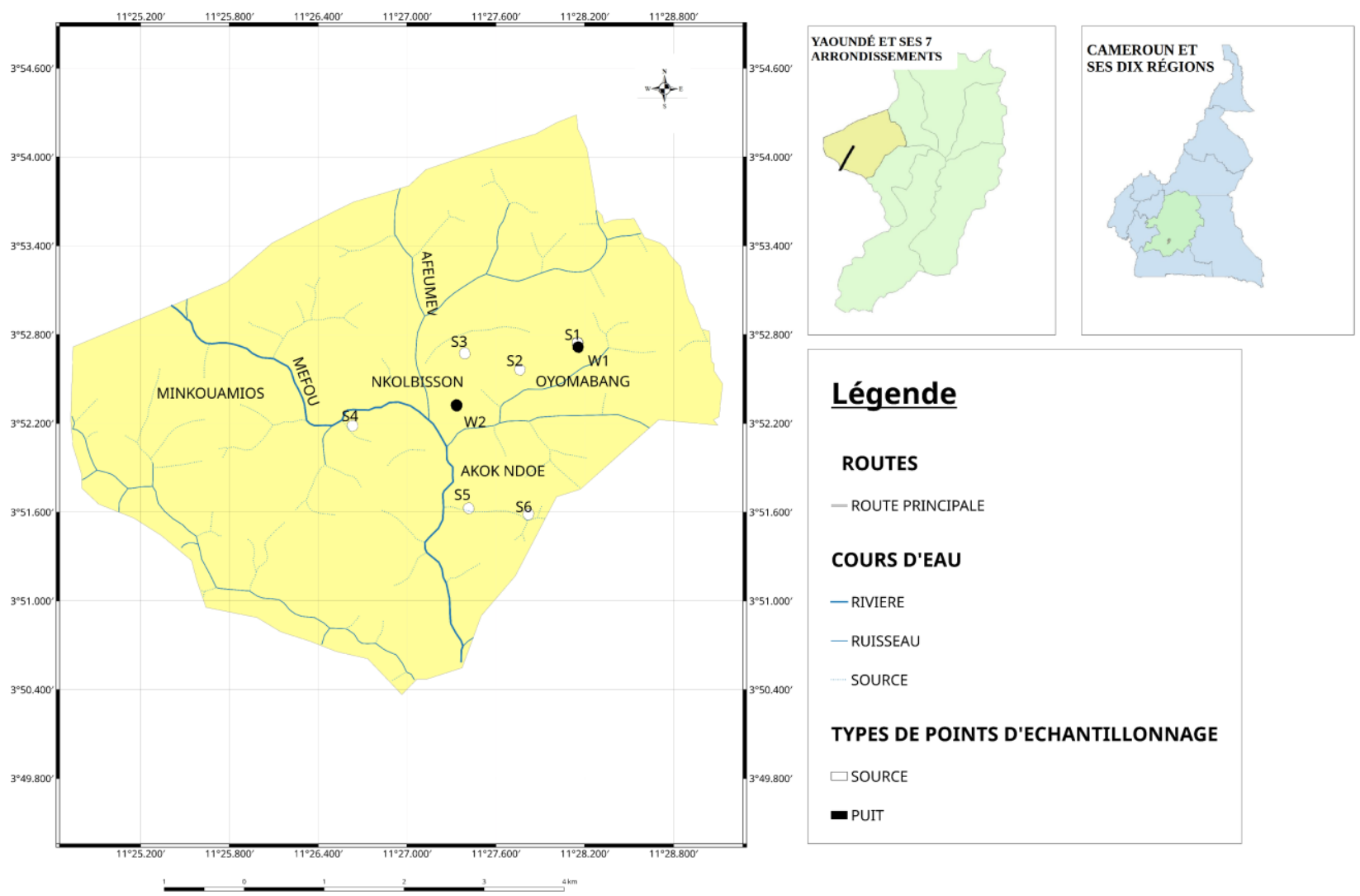

Figure 1: Localisation des stations d'échantillonnage sur le site d'étude.

Source: Carte topographique Yaoundé 3d. NA-32XXIV à l'échelle 1/50 000 dessinée par IGN Paris, 1956, digitalisée par Diane MOUSSIMA, 2019.

Tableau 1 : Description des sites d'étude.

\begin{tabular}{|c|c|c|c|c|c|}
\hline \multirow[t]{2}{*}{ Sites } & \multirow[t]{2}{*}{ Quartiers } & \multirow[t]{2}{*}{ Caractéristiques } & \multicolumn{3}{|c|}{ Coordonnés géographiques } \\
\hline & & & Latitude & Longitude & Altitude \\
\hline Source 1 (S1) & Oyom Abang & $\begin{array}{l}\text { Aménagée, proximité } \\
\text { des habitations }\end{array}$ & $\mathrm{N} 03^{\circ} 52^{\prime} 44.7^{\prime \prime}$ & E $011^{\circ} 28^{\prime} 09.2^{\prime \prime}$ & $723 \mathrm{~m}$ \\
\hline Source 2 (S2) & Oyom Abang & $\begin{array}{l}\text { Aménagée, proximité } \\
\text { des habitations }\end{array}$ & $\mathrm{N} 03^{\circ} 52^{\prime} 33.8^{\prime \prime}$ & E $011^{\circ} 27^{\prime} 45.8^{\prime \prime}$ & $706 \mathrm{~m}$ \\
\hline Source 3 (S3) & Nkolbisson & $\begin{array}{l}\text { Aménagée, proximité } \\
\text { des habitations }\end{array}$ & $\mathrm{N} 03^{\circ} 52^{\prime} 40.2^{\prime}$ & E $011^{\circ} 27^{\prime} 23.4^{\prime \prime}$ & $718 \mathrm{~m}$ \\
\hline Source 4 (S4) & Nkolbisson & $\begin{array}{l}\text { Aménagée, proximité } \\
\text { des habitations }\end{array}$ & $\mathrm{N} 03^{\circ} 52^{\prime} 11.0^{\prime \prime}$ & E $011^{\circ} 26^{\prime} 37.9^{\prime \prime}$ & $706 \mathrm{~m}$ \\
\hline Source 5 (S5) & Akok Ndoé & $\begin{array}{lr}\text { Non } & \text { aménagée, } \\
\text { proximité } & \text { des }\end{array}$ & N $03^{\circ} 51^{\prime} 37.6^{\prime \prime}$ & E $011^{\circ} 27^{\prime} 25.0^{\prime \prime}$ & $712 \mathrm{~m}$ \\
\hline
\end{tabular}


habitations

\begin{tabular}{|c|c|c|c|c|c|}
\hline Source 6 (S6) & Akok Ndoé & $\begin{array}{l}\text { Non aménagée, loin } \\
\text { des habitations }\end{array}$ & $\mathrm{N} 03^{\circ} 51^{\prime} 35.2^{\prime \prime}$ & E $011^{\circ} 27^{\prime} 49.1^{\prime \prime}$ & $740 \mathrm{~m}$ \\
\hline Puits 1 (W1) & Oyom Abang & $\begin{array}{l}\text { Hauteur } \\
0.3 \mathrm{~m}, \\
\text { proximité } \\
\text { habitations }\end{array}$ & $\mathrm{N} 03^{\circ} 52^{\prime} 42.9^{\prime \prime}$ & E $011^{\circ} 28^{\prime} 09.4^{\prime \prime}$ & $723 \mathrm{~m}$ \\
\hline Puits 2 (W2) & Nkolbisson & $\begin{array}{lr}\text { Hauteur } & \text { margelle } \\
0.5 \mathrm{~m}, \text { non } & \text { couvert, } \\
\text { proximité } & \text { des } \\
\text { habitations } & \end{array}$ & N 0352’19.2" & E $011^{\circ} 27^{\prime} 20.2^{\prime \prime}$ & $704 \mathrm{~m}$ \\
\hline
\end{tabular}

Tableau 2 : Méthodes d'analyse des eaux échantillonnées.

\begin{tabular}{|c|c|c|c|c|}
\hline Paramètre & Technique & Site de mesure & Appareil & Méthode \\
\hline Température $\left({ }^{\circ} \mathrm{C}\right)$ & Lecture Directe & In situ & $\begin{array}{l}\text { Multiparamètre de marque } \\
\text { Thermo-scientific ORION } \\
\text { STAR } 225\end{array}$ & NF ISO 10523, \\
\hline PH (UC) & Lecture Directe & In situ & $\begin{array}{l}\text { Multiparamètre de marque } \\
\text { Thermo-scientific ORION } \\
\text { STAR } 225\end{array}$ & NF ISO 10523, \\
\hline $\begin{array}{l}\text { conductivité électrique } \\
(\mu \mathrm{S} / \mathrm{cm}) \text { et TDS }(\mathrm{mg} / \mathrm{l})\end{array}$ & Lecture Directe & In situ & $\begin{array}{l}\text { Multiparamètre de marque } \\
\text { Thermo-scientific ORION } \\
\text { STAR } 225\end{array}$ & NF ISO 10523, \\
\hline $\begin{array}{l}\text { Bicarbonate }(\mathrm{mg} / \mathrm{l}) \text { et } \\
\text { carbonate }(\mathrm{mg} / \mathrm{l})\end{array}$ & $\begin{array}{l}0.25 \mathrm{~N} \\
\text { Titration }\end{array}$ & In labo & Burette graduée & NF EN ISO9963-1 \\
\hline Sulfates (mg/l) & $\begin{array}{l}\text { Baryum chromate } \\
\text { Gravimétrie }\end{array}$ & In labo & $\begin{array}{l}\text { Spectrophotomètre } \\
\text { marque jenway } 206\end{array}$ & NF ISO 11048:1995 \\
\hline Phosphates (mg/l) & $\begin{array}{l}\text { Molybate } \\
\text { Colorimétrie }\end{array}$ & In labo & $\begin{array}{l}\text { Spectrophotomètre } \\
\text { marque jenway } 206\end{array}$ & NF ISO 6878: 2004 \\
\hline Chlore (mg/l) & $\begin{array}{l}\text { Sulfate d'argent } \\
\text { Titration }\end{array}$ & In labo & Burette graduée & NF ISO 9297 \\
\hline Nitrate (mg/l) & $\begin{array}{l}\text { Acide } \\
\text { Sulphosalicylique } \\
\text { Colorimétrie }\end{array}$ & In labo & $\begin{array}{l}\text { Spectrophotometre } \\
\text { marque jenway } 206\end{array}$ & NF ISO 7890-3: 1988 \\
\hline $\begin{array}{l}\text { Sodium, Potassium, } \\
\text { Calcium, Magnésium } \\
(\mathrm{mg} / \mathrm{l})\end{array}$ & Lecture Directe & In labo & $\begin{array}{l}\text { ICP OES de marque } \\
\text { Optima } 8000\end{array}$ & NF ISO 11885 \\
\hline
\end{tabular}




\section{RÉSULTATS \\ Qualité des eaux \\ Qualité physico-chimique des eaux}

Le Tableau 3 présente les valeurs minimales (min), médianes (med), moyennes (moy) et maximales (max) des résultats des analyses physico-chimiques obtenus à chaque station ainsi que leur pourcentage de nonconformité par rapport à la réglementation nationale en matière de potabilité de l'eau de consommation (NC 207, 2014). Les valeurs des médianes des paramètres température, $\mathrm{pH}$, conductivité électrique, TDS, bicarbonates, calcium, magnésium sodium et potassium, sont restées proches de leurs valeurs moyennes. Les plus fortes valeurs de température ont été enregistrées à $\mathrm{W}_{1}$ (moy : $26.29^{\circ} \mathrm{C}$, med : $26.4^{\circ} \mathrm{C}$ ) tandis que les plus faibles valeurs ont été lu à $\mathrm{S}_{6}$ (moy : $23.74{ }^{\circ} \mathrm{C}$; med : $23.63{ }^{\circ} \mathrm{C}$ ). Les eaux de la station $\mathrm{S}_{2}$ avec une moyenne de 3.90 et une médiane de 3.94 ont été les plus acides. Les valeurs maximales de $\mathrm{pH}$ ont été lu à $\mathrm{S}_{6}$ (moy : 5.90; med : 5.88). Les concentrations des ions orthophosphates et sulfates dans les eaux échantillonnées sont inférieures à la limite de détection du spectrophotomètre utilisé. Les eaux des stations $\mathrm{S}_{1}, \mathrm{~W}_{1}$ et $\mathrm{W}_{2}$ sont les plus concentrées en ions nitrates (moy:48.12 mg/l, med:36.06 mg/l; moy: $41.61 \mathrm{mg} / 1$, med:28.21 $\mathrm{mg} / \mathrm{l}$; moy:78.53 mg/l ,med: $80.70 \mathrm{mg} / \mathrm{l}$ respectivement pour $\mathrm{S}_{1}, \mathrm{~W}_{1}$ et $\mathrm{W}_{2}$ ), chlorures (moy:16.62 mg/l, med:15.45 mg/l; moy:35.46 mg/l, med: $34.94 \mathrm{mg} / 1$; moy: $22.72 \mathrm{mg} / 1$, med: $21.93 \mathrm{mg} / \mathrm{l}$ respectivement pour $\mathrm{S}_{1}, \mathrm{~W}_{1}$ et $\mathrm{W}_{2}$ ), sodium (moy:22.898 mg/l, med:23.127 mg/l; moy:45.693 mg/l, med:46.355 mg/l; moy:30.326 mg/l, med:30.253 mg/l respectivement pour $\mathrm{S}_{1}, \mathrm{~W}_{1}$ et $\mathrm{W}_{2}$ ) et potassium (moy:5.345 mg/l, med:5.357 mg/l; moy:10.704 mg/l, med:10.550 mg/l; moy:10.897 mg/l, med:10.857 mg/l respectivement pour $\mathrm{S}_{1}, \mathrm{~W}_{1}$ et $\mathrm{W}_{2}$ ). Elles ont également obtenu les plus fortes valeurs de conductivité électrique (moy : $137.25 \mu \mathrm{S} / \mathrm{cm}$, med : $133.68 \mu \mathrm{S} / \mathrm{cm}$; moy : $261.55 \mu \mathrm{S} / \mathrm{cm}$. med : $256.13 \mu \mathrm{S} / \mathrm{cm}$; moy : $204.92 \mu \mathrm{S} / \mathrm{cm}$, med : $204.60 \mu \mathrm{S} / \mathrm{cm}$ respectivement pour $S_{1}$, $\mathrm{W}_{1}$ et $\mathrm{W}_{2}$ ). Concernant la dureté, les eaux des puits ont obtenu les plus fortes teneurs en ions calcium (moy : $3.809 \mathrm{mg} / \mathrm{l}$, med : $3.779 \mathrm{mg} / \mathrm{l}$; moy : $5.953 \mathrm{mg} / \mathrm{l}$ med : $5.952 \mathrm{mg} / \mathrm{l}$ respectivement pour $\mathrm{W}_{1}$ et $\mathrm{W}_{2}$ ) et magnésium (moy : $1.818 \mathrm{mg} / \mathrm{l}$, med : $1.809 \mathrm{mg} / \mathrm{l}$; moy : $2.970 \mathrm{mg} / \mathrm{l}$, med: $2.981 \mathrm{mg} / \mathrm{l}$ pour $\mathrm{W}_{1}$ et $\mathrm{W}_{2}$ respectivement). Le taux de non-conformité par rapport à la NC 207 (2014) du pH a été de $100 \%$ à pratiquement toutes les stations excepté $\mathrm{S}_{6}$ où il est de $33.33 \%(6,5-8$,$) . Les$ teneurs des eaux des stations $\mathrm{S}_{1}, \mathrm{~W}_{1}$ et $\mathrm{W}_{2}$ en ions nitrate supérieures à $50 \mathrm{mg} / \mathrm{l}$ sont en marge des limites recommandées par la NC 207 (2014), avec des taux de non conformités respectifs de $33.33 \%, 33.33 \%$ et $100 \%$.

\section{Qualité bactériologique des eaux}

Les stations $\mathrm{S}_{5}, \mathrm{~S}_{6}, \mathrm{~W}_{1}$ et $\mathrm{W}_{2}$ présentent les plus fortes valeurs de la flore aérobie mésophile totale (moy : $10 \mathrm{ufc} / \mathrm{ml}$, med : 3 ufc/ml ; moy : $22 \mathrm{ufc} / \mathrm{ml}$, med : $17 \mathrm{ufc} / \mathrm{ml}$; moy: $65 \mathrm{ufc} / \mathrm{ml}$, med: $61 \mathrm{ufc} / \mathrm{ml}$; moy:199 $\mathrm{ufc} / \mathrm{ml}$, med:100 ufc/ml respectivement pour $\mathrm{S}_{1}, \mathrm{~S}_{6}, \mathrm{~W}_{1}$ et $\mathrm{W}_{2}$ ). Pour ce paramètre, elles présentent des taux de conformité respectifs de $33.33 \%, 33.33 \%, 100 \%$ et $100 \%$. Les Entérocoques intestinaux ont été retrouvés une campagne sur trois dans les eaux des stations $\mathrm{S}_{2}, \mathrm{~S}_{3}$, et $\mathrm{S}_{4}$ (taux de non-conformité de $33.33 \%$ ), deux campagnes sur trois dans les eaux de $\mathrm{S}_{4}, \mathrm{~S}_{5}$ et $\mathrm{W}_{2}$ (taux de non-conformité $66.67 \%$ ), à toutes les campagnes dans les eaux de $\mathrm{W}_{1}$ (taux de non-conformité $100 \%$ ) et pas une seule fois dans les eaux de $S_{1}$ Figure 2. Les Streptocoques fécaux, quant à eux, ont été absents dans les eaux de $S_{1}$ et $S_{2}$ (taux de nonconformité $0 \%$ ), présents une campagne sur 3 dans les eaux de $\mathrm{S}_{3}, \mathrm{~S}_{6}$, et $\mathrm{W}_{1}$ (taux de nonconformité $33.33 \%$ ) et deux sur trois dans les eaux de $\mathrm{S}_{4}, \mathrm{~S}_{5}$ et $\mathrm{W}_{2}$ (Figure 3 ). Les salmonelles ont été identifiées à toutes les stations. Les fréquences d'apparition étant respectivement de $33.33 \%$ à $\mathrm{S}_{1}$ et $\mathrm{S}_{2}$, de $66.67 \%$ à $\mathrm{W}_{1}, \mathrm{~W}_{2}$ et de $100 \%$ à $\mathrm{S}_{3}, \mathrm{~S}_{4}, \mathrm{~S}_{6}$ et $\mathrm{S}_{5}$ (Figure 4). 


\section{Faciès chimique des eaux des puits et des sources}

Le diagramme de Piper (Figure 5), présente une classification des puits et sources étudiés suivant les faciès chimiques de leurs eaux. L'analyse globale des 24 échantillons représentés dans le losange regroupe les eaux de $66 \%$ de sources et $100 \%$ de puits dans la famille chimique chlorurées sodiques et potassiques. Les eaux de $17 \%$ de sources montrent un faciès chloruré et sulfaté calcique et magnésique. Les eaux des sources restantes (17\%) sont bicarbonatées calciques et magnésiques. Le triangle des cations révèle une dominance des ions sodium et potassium. Dans le triangle des anions, les eaux échantillonnées se regroupent dans le pole chlorures et nitrates.

\section{Corrélations}

La Figure 6 illustre les corrélations qui existent entre les paramètres bactériologiques, physique et chimiques. Elle présente graphiquement les relations entre ces variables (courbes de régression). Elle montre également, à travers les histogrammes affichés sur la diagonale, la distribution de chaque variable. Les variables très fortement corrélées présentent des distributions quasi semblables. Il $\mathrm{y}^{\prime}$ a une forte corrélation négative entre la température et le $\mathrm{pH}(\mathrm{r}=-$ 0.60). Le $\mathrm{pH}$ est d'autre part significativement corrélé aux ions bicarbonates $(\mathrm{r}=0.51)$. La FAMT est significativement corrélée aux ions sodium $(\mathrm{r}=0.45)$, chlorures $(\mathrm{r}=0.44)$ ainsi qu'à la conductivité électrique $(\mathrm{r}=0.50)$. Elle est fortement corrélée aux ions nitrates $(r=0.53)$ et potassium $(\mathrm{r}=0.61)$. Ce paramètre est également très fortement corrélé aux ions calcium $(r=0.70)$. La conductivité électrique est très fortement corrélée aux TDS, nitrates, chlorures, calcium, sodium et potassium $(\mathrm{r}=1.00 ; \mathrm{r}=0.64 ; \mathrm{r}=0.98 ; \mathrm{r}=0.79 ; \mathrm{r}=0.99$; $\mathrm{r}=0.97$ respectivement). Elle est également fortement corrélée aux ions bicarbonates $(\mathrm{r}=0.61)$. Les ions nitrates sont fortement corrélés aux ions chlorures $(r=0.60)$ et, aux ions sodiums ( $\mathrm{r}=0.58)$. Les ions chlorures sont très fortement corrélés aux ions bicarbonates $(r=0.66)$, calcium $(r=0.71)$, sodium $(r=0.98)$ et potassium $(r=0.94)$. Le potassium est très fortement corrélé au calcium $(\mathrm{r}=0.87)$, et au sodium $(r=0.95)$.

\section{Catégorisation des stations échantillonnage}

L'analyse en composantes principales (ACP) groupe les variables analysées en de nouvelles. Celles-ci forment des axes. L'axe 1 des abscisses de la Figure $7 b$, regroupe $45.38 \%$ de données et est principalement formé des variables nitrates, calcium, FAMT, dureté totale. Cet axe qui regroupe des éléments de pollution sera renomme axe de pollution. L'axe 2 des ordonnées regroupe $25.1 \%$ des données et est essentiellement formé des paramètres $\mathrm{pH}$ et bicarbonates. Il sera donc renommé axe de l'acidité.

En transposant ces axes sur la Figure $7 b$ il ressort que $W_{2}$ est caractérisé par des eaux plus polluées et acides que les autres. $\mathrm{W}_{1}$ est caractérise par des eaux polluées et moins acides que les autres. La station $\mathrm{S}_{6}$ se démarque également par des eaux moins polluées et relativement moins acides tandis que les eaux des stations $\mathrm{S}_{1}, \mathrm{~S}_{2}, \mathrm{~S}_{3}, \mathrm{~S}_{4}$ et $\mathrm{S}_{5}$ forment un groupe dont les eaux sont relativement moins polluées et relativement acides.

La méthode de classification ascendante hiérarchique permet de définir les similitudes entre les stations échantillonnées. Ce dendrogramme (Figure 8) présente 5 classes de stations d'échantillonnage. $S_{1}, S_{2}$, $\mathrm{S}_{3}, \mathrm{~S}_{4}$ et $\mathrm{S}_{5}$ définies par l'ACP (Figure 7) comme étant un seul groupe, a été éclaté en deux classes. La classe 2 contenant $S_{1}$ et $S_{2}$ et, la classe 3 contenant $S_{3}, S_{4}$ et $S_{5}$. Les classes sont donc les suivantes :

$>\quad$ Classe 1 contenant tous les échantillons de $\mathrm{S}_{6}$ et l'échantillon $\mathrm{S}_{5 \mathrm{c}}$. Ces eaux sont caractérisées par leur légère acidité (moy $\mathrm{pH}=5.88$ et $\mathrm{pH}=5.84$ respectivement pour $\mathrm{S}_{6}$ et $\mathrm{S}_{5 \mathrm{C}}$ ), et la présence de la 
FAMT $($ moy $=27 \mathrm{ufc} / \mathrm{ml}$ et $41 \mathrm{ufc} / \mathrm{ml}$ respectivement pour $\mathrm{S}_{6}$ et $\mathrm{S}_{5 \mathrm{C}}$ ).

$>$ Classe 2 comprenant les stations $S_{1}$ et $S_{2}$ caractérisées par des teneurs en nitrate concentrées autour de la trentaine (med=36.06 $\mathrm{mg} / \mathrm{l}$ et $32.4 \mathrm{mg} / \mathrm{l}$ respectivement pour $S_{1}$ et $S_{2}$ ) et l'absence de FAMT

Classe 3 constituée de $S_{3}, S_{4}, S_{5 A}$ et $S_{5 B}$. Leurs eaux sont dépourvues de FAMT et sont un peu plus acides que celle des stations de la classe $1 \quad$ (moy $=4.46$, med $=4.38 ;$ moy $=4.49$, med $=4.49 ; 4.17$ et 4.00 respectivement pour $\mathrm{S}_{3}, \mathrm{~S}_{4}, \mathrm{~S}_{5 \mathrm{~A}}$ et $\left.\mathrm{S}_{5 \mathrm{~B}}\right)$.
Classe 4 contient les échantillons d'eau de $\mathrm{W}_{1}$ caractérisées par un $\mathrm{pH}$ moyen plus élevé que tous les autres (moy $=5.20$, med=5.20), une teneur en nitrate qui se démarque de celle des eaux de source ( $\mathrm{moy}=41.61 \mathrm{mg} / \mathrm{l}$, med=28.21 mg/l) et une concentration assez forte en FAMT ( $\mathrm{moy}=65 \mathrm{ufc} / \mathrm{ml}$, med=61 ufc $/ \mathrm{ml}$ ).

$>$ Classe 5 des eaux du puit $\mathrm{W}_{2}$. Ces eaux sont les plus acides (moy $=4.45$, med $=4.35)$, riches en nitrates (moy=78.53 mg/l, med=80.70) et en FAMT $\quad(\operatorname{moy}=199 \mathrm{ufc} / \mathrm{ml}, \quad \mathrm{med}=100$ ufc/l).

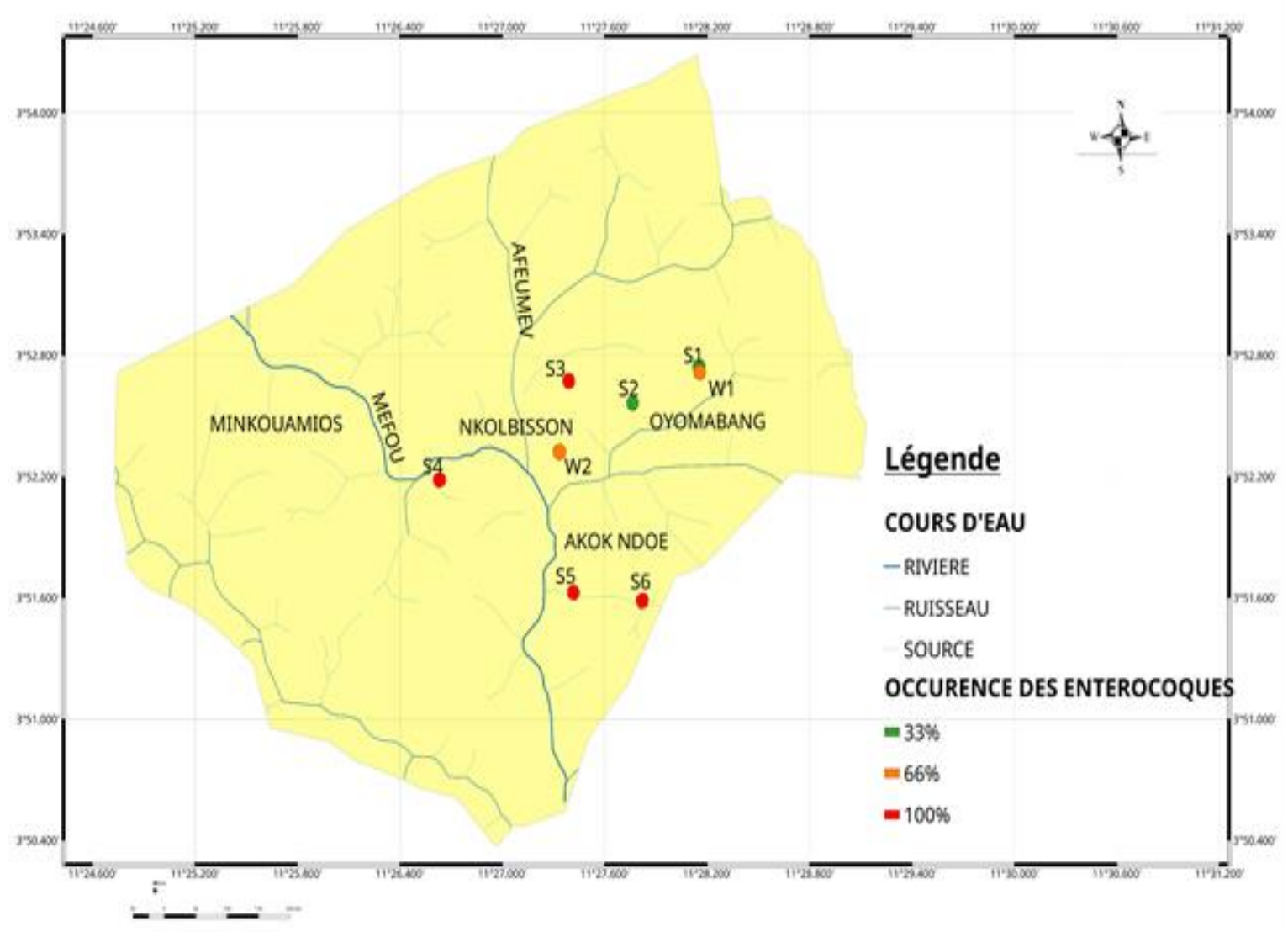

Figure 2 : Distribution des entérocoques intestinaux dans la zone d'étude.

Source: Carte topographique Yaoundé 3d. NA-32XXIV à l'échelle 1/50 000 dessinée par IGN Paris, 1956, digitalisée par Diane MOUSSIMA, 2019. 
Tableau 3: Propriétés physico-chimiques des eaux.

\begin{tabular}{|c|c|c|c|c|c|c|c|c|c|c|c|c|c|c|c|}
\hline Paramètre & $\mathbf{N}$ & $\mathbf{T}^{\circ} \mathbf{C}$ & $\begin{array}{r}\mathbf{p H} \\
(\mathbf{U C})\end{array}$ & $\begin{array}{r}\mathrm{CE} \\
(\mu \mathrm{S} / \mathrm{cm})\end{array}$ & $\begin{array}{r}\text { TDS } \\
(\mathrm{mg} / \mathrm{l})\end{array}$ & $\begin{array}{l}\text { PO43- } \\
(\mathrm{mg} / \mathrm{l})\end{array}$ & $\begin{array}{l}\text { SO42- } \\
(\mathrm{mg} / \mathrm{l})\end{array}$ & $\begin{array}{l}\text { NO3- } \\
(\mathrm{mg} / \mathrm{l})\end{array}$ & $\begin{array}{r}\mathrm{Cl}- \\
(\mathrm{mg} / \mathrm{l})\end{array}$ & $\begin{array}{r}\text { HCO3- } \\
(\mathrm{mg} / \mathrm{l})\end{array}$ & $\begin{array}{r}\mathrm{Ca} 2+ \\
(\mathrm{mg} / \mathrm{l})\end{array}$ & $\begin{array}{l}\mathrm{Mg} 2+ \\
(\mathrm{mg} / \mathrm{l})\end{array}$ & $\begin{array}{r}\text { Durete } \\
\text { (of) }\end{array}$ & $\begin{array}{r}\mathrm{Na}+ \\
(\mathrm{mg} / \mathrm{l})\end{array}$ & $\begin{array}{r}\mathrm{K}+ \\
(\mathrm{mg} / \mathrm{l})\end{array}$ \\
\hline $\begin{array}{l}\text { valeurs limites OMS } \\
\text { (2011) }\end{array}$ & l & 25 & $6,5-9,2$ & $500-1400$ & l & / & 500 & 50 & 2502 & $200-300$ & 200 & 150 & & 200 & 100 \\
\hline $\begin{array}{l}\text { Norme Camerounaise } \\
\text { NC } 207 \text { (NC, 2014) }\end{array}$ & l & I & $6.5-8$ & I & l & 5 & 250 & 50 & 250 & / & l & 50 & / & 150 & 12 \\
\hline S1 Min & & 24.47 & 4.56 & 133.65 & 138.7 & $\mathrm{dl}$ & $\mathrm{dl}$ & 17.83 & 13.40 & 12.49 & 1.355 & 13.40 & 0.917 & 22.390 & 5.276 \\
\hline Med & 3 & 24.56 & 4.58 & 133.68 & 140.0 & $\mathrm{dl}$ & $\mathrm{dl}$ & 36.06 & 15.45 & 12.51 & 1.363 & 15.45 & 0.917 & 23.127 & 5.357 \\
\hline Moy & & 24.85 & 4.58 & 137.25 & 141.6 & $\mathrm{dl}$ & $\mathrm{dl}$ & 48.12 & 16.62 & 12.81 & 1.366 & 16.62 & 0.930 & 22.898 & 5.345 \\
\hline $\operatorname{Max}$ & & 25.51 & 4.60 & 144.41 & 146.0 & $\mathrm{dl}$ & $\mathrm{dl}$ & 90.48 & 21.00 & 13.42 & 1.380 & 21.00 & 0.956 & 23.177 & 5.403 \\
\hline$\%$ non conformité & & I & 100 & I & I & 0 & 0 & 33.33 & 0 & I & 0 & 0 & I & 0 & 0 \\
\hline S2 Min & & 24.81 & 3.78 & 64.52 & 64.7 & $\mathrm{dl}$ & $\mathrm{dl}$ & 28.22 & 3.02 & 0.00 & 1.570 & 1.383 & 0.969 & 6.837 & 1.963 \\
\hline Med & 3 & 24.85 & 3.94 & 67.46 & 70.0 & $\mathrm{dl}$ & $\mathrm{dl}$ & 32.41 & 4.74 & 4.19 & 1.643 & 1.403 & 0.996 & 7.644 & 1.963 \\
\hline Moy & & 25.54 & 3.90 & 68.12 & 70.2 & $\mathrm{dl}$ & $\mathrm{dl}$ & 32.10 & 5.00 & 4.72 & 1.679 & 1.401 & 1.004 & 7.728 & 2.032 \\
\hline $\operatorname{Max}$ & & 26.98 & 3.97 & 72.38 & 76.0 & $\mathrm{dl}$ & $\mathrm{dl}$ & 35.68 & 7.23 & 9.98 & 1.823 & 1.417 & 1.046 & 8.703 & 2.170 \\
\hline$\%$ non conformité & & I & 100 & I & I & 0 & 0 & 0 & 0 & I & 0 & 0 & I & 0 & 0 \\
\hline S3 Min & & 23.91 & 4.26 & 74.57 & 74.7 & $\mathrm{dl}$ & $\mathrm{dl}$ & 10.56 & 3.18 & 12.86 & 1.477 & 3.649 & 1.922 & 7.718 & 2.048 \\
\hline Med & 3 & 25.21 & 4.38 & 76.57 & 78.0 & $\mathrm{dl}$ & $\mathrm{dl}$ & 17.53 & 3.63 & 13.27 & 1.540 & 3.726 & 1.946 & 8.023 & 2.084 \\
\hline Moy & & 24.79 & 4.46 & 77.22 & 78.6 & $\mathrm{dl}$ & $\mathrm{dl}$ & 19.54 & 4.56 & 13.22 & 1.590 & 3.707 & 1.942 & 7.961 & 2.097 \\
\hline $\operatorname{Max}$ & & 25.26 & 4.74 & 80.53 & 83.0 & $\mathrm{dl}$ & $\mathrm{dl}$ & 30.52 & 6.89 & 13.52 & 1.753 & 3.746 & 1.959 & 8.143 & 2.159 \\
\hline$\%$ non conformité & & I & 100 & / & / & 0 & 0 & 0 & 0 & I & 0 & 0 & I & 0 & 0 \\
\hline
\end{tabular}


D. A. MOUSSIMA YAKA et al. / Int. J. Biol. Chem. Sci. 14(5): 1902-1920, 2020

\begin{tabular}{|c|c|c|c|c|c|c|c|c|c|c|c|c|c|c|c|}
\hline S4 Min & & 24.20 & 4.45 & 69.27 & 70.3 & dl & $\mathrm{dl}$ & 10.42 & 2.79 & 13.12 & 1.907 & 2.798 & 1.645 & 7.776 & 1.777 \\
\hline Med & 3 & 25.63 & 4.49 & 70.47 & 72.0 & $\mathrm{dl}$ & $\mathrm{dl}$ & 12.66 & 3.14 & 13.74 & 1.973 & 2.804 & 1.662 & 7.953 & 1.844 \\
\hline Moy & & 25.44 & 4.49 & 71.09 & 72.6 & $\mathrm{dl}$ & $\mathrm{dl}$ & 23.93 & 4.45 & 14.22 & 2.120 & 2.802 & 1.697 & 7.907 & 1.867 \\
\hline Max & & 26.50 & 4.54 & 73.55 & 75.3 & $\mathrm{dl}$ & $\mathrm{dl}$ & 48.70 & 7.43 & 15.81 & 2.480 & 2.804 & 1.786 & 7.993 & 1.981 \\
\hline$\%$ non conformité & & I & 100 & I & I & 0 & 0 & 0 & 0 & I & 0 & 0 & I & 0 & 0 \\
\hline S5 Min & & 24.57 & 4.00 & 48.87 & 50.0 & $\mathrm{dl}$ & $\mathrm{dl}$ & 1.87 & $\mathrm{dl}$ & 11.85 & 1.254 & 2.379 & 1.369 & 4.477 & 1.086 \\
\hline Med & 3 & 24.67 & 4.17 & 48.90 & 50.7 & $\mathrm{dl}$ & $\mathrm{dl}$ & 14.16 & 1.00 & 11.87 & 1.604 & 2.533 & 1.392 & 4.531 & 1.119 \\
\hline Moy & & 24.64 & 4.67 & 50.33 & 51.7 & $\mathrm{dl}$ & $\mathrm{dl}$ & 13.08 & 1.82 & 12.06 & 1.723 & 2.505 & 1.475 & 4.643 & 1.200 \\
\hline Max & & 24.70 & 5.84 & 53.23 & 54.33 & $\mathrm{dl}$ & $\mathrm{dl}$ & 23.33 & 4.46 & 12.46 & 2.312 & 2.604 & 1.663 & 4.920 & 1.394 \\
\hline$\%$ non conformité & & I & 100 & I & I & 0 & 0 & 0 & 0 & I & 0 & 0 & I & 0 & 0 \\
\hline S6 Min & & 23.07 & 5.09 & 22.63 & 23.0 & $\mathrm{dl}$ & $\mathrm{dl}$ & $\mathrm{dl}$ & $\mathrm{dl}$ & 15.46 & 0.426 & 1.849 & 0.877 & 1.565 & 0.573 \\
\hline Med & 3 & 23.63 & 5.90 & 22.87 & 23.00 & $\mathrm{dl}$ & $\mathrm{dl}$ & 8.60 & 0.98 & 16.84 & 0.46 & 1.89 & 0.90 & 1.57 & 0.68 \\
\hline Moy & & 23.74 & 5.88 & 23.11 & 23.3 & $\mathrm{dl}$ & $\mathrm{dl}$ & 10.37 & 1.26 & 17.80 & 0.481 & 1.877 & 0.902 & 1.566 & 0.664 \\
\hline $\operatorname{Max}$ & & 24.53 & 6.66 & 23.83 & 24.0 & $\mathrm{dl}$ & $\mathrm{dl}$ & 22.50 & 2.80 & 21.09 & 0.551 & 1.896 & 0.928 & 1.567 & 0.742 \\
\hline$\%$ non conformité & & I & 33.33 & I & / & 0 & 0 & 0 & 0 & I & 0 & 0 & I & 0 & 0 \\
\hline W1 Min & & 23.77 & 5.01 & 246.47 & 264.7 & dl & $\mathrm{dl}$ & 25.69 & 33.18 & 37.30 & 3.763 & 2.008 & 1.777 & 44.256 & 10.186 \\
\hline Med & 3 & 23.80 & 5.06 & 256.13 & 271.7 & $\mathrm{dl}$ & $\mathrm{dl}$ & 28.21 & 34.94 & 38.56 & 3.779 & 2.075 & 1.809 & 46.355 & 10.550 \\
\hline Moy & & 24.09 & 5.20 & 261.55 & 273.3 & $\mathrm{dl}$ & $\mathrm{dl}$ & 41.61 & 35.46 & 38.61 & 3.809 & 2.078 & 1.818 & 45.693 & 10.704 \\
\hline $\operatorname{Max}$ & & 24.70 & 5.53 & 282.04 & 283.7 & $\mathrm{dl}$ & $\mathrm{dl}$ & 70.95 & 38.27 & 39.96 & 3.884 & 2.152 & 1.867 & 46.469 & 11.377 \\
\hline$\%$ non conformité & & I & 100 & l & I & 0 & 0 & 33.33 & 0 & I & 0 & 0 & I & 0 & 0 \\
\hline W2 Min & & 25.20 & 4.24 & 202.67 & 205.0 & $\mathrm{dl}$ & $\mathrm{dl}$ & 66.90 & 21.89 & 9.26 & 5.952 & 3.446 & 2.925 & 29.490 & 10.857 \\
\hline Med & 3 & 26.40 & 4.35 & 204.60 & 206.3 & $\mathrm{dl}$ & $\mathrm{dl}$ & 80.70 & 21.93 & 10.82 & 5.952 & 3.583 & 2.981 & 30.253 & 10.857 \\
\hline Moy & & 26.29 & 4.45 & 204.92 & 208.2 & $\mathrm{dl}$ & $\mathrm{dl}$ & 78.53 & 22.72 & 11.39 & 5.953 & 3.556 & 2.970 & 30.326 & 10.897 \\
\hline $\operatorname{Max}$ & & 27.27 & 4.75 & 207.5 & 213.3 & $\mathrm{dl}$ & $\mathrm{dl}$ & 87.98 & 24.34 & 14.09 & 5.956 & 3.639 & 3.004 & 31.233 & 10.977 \\
\hline$\%$ non conformité & & I & 100 & I & I & 0 & 0 & 100 & 0 & I & 0 & 0 & I & 0 & 0 \\
\hline
\end{tabular}




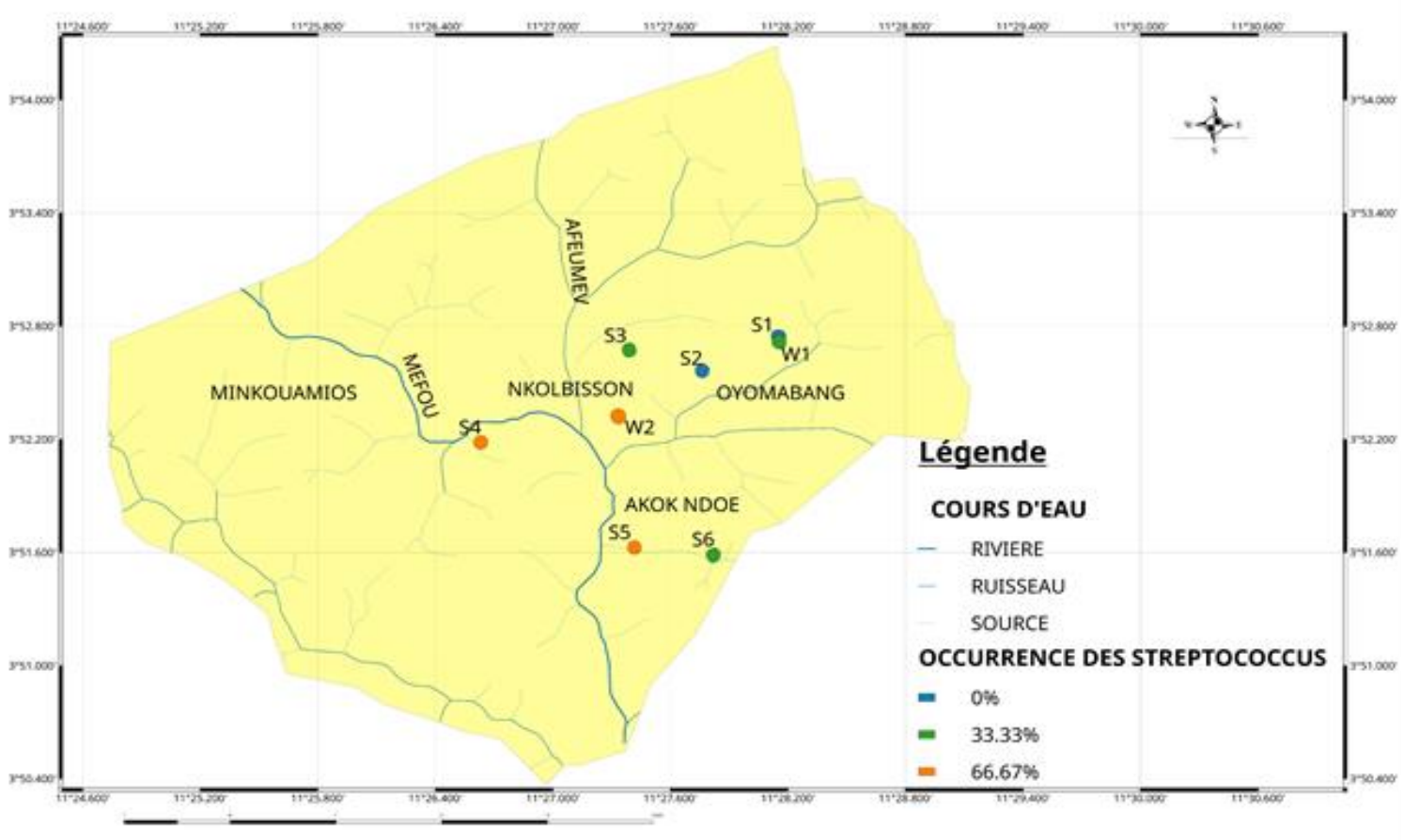

Figure 3 : Distribution des streptocoques fécaux dans la zone d'étude.

Source: carte topographique Yaoundé 3d. NA-32XXIV à l'échelle 1/50 000 dessinée par IGN Paris, 1956, digitalisée par Diane MOUSSIMA, 2019.

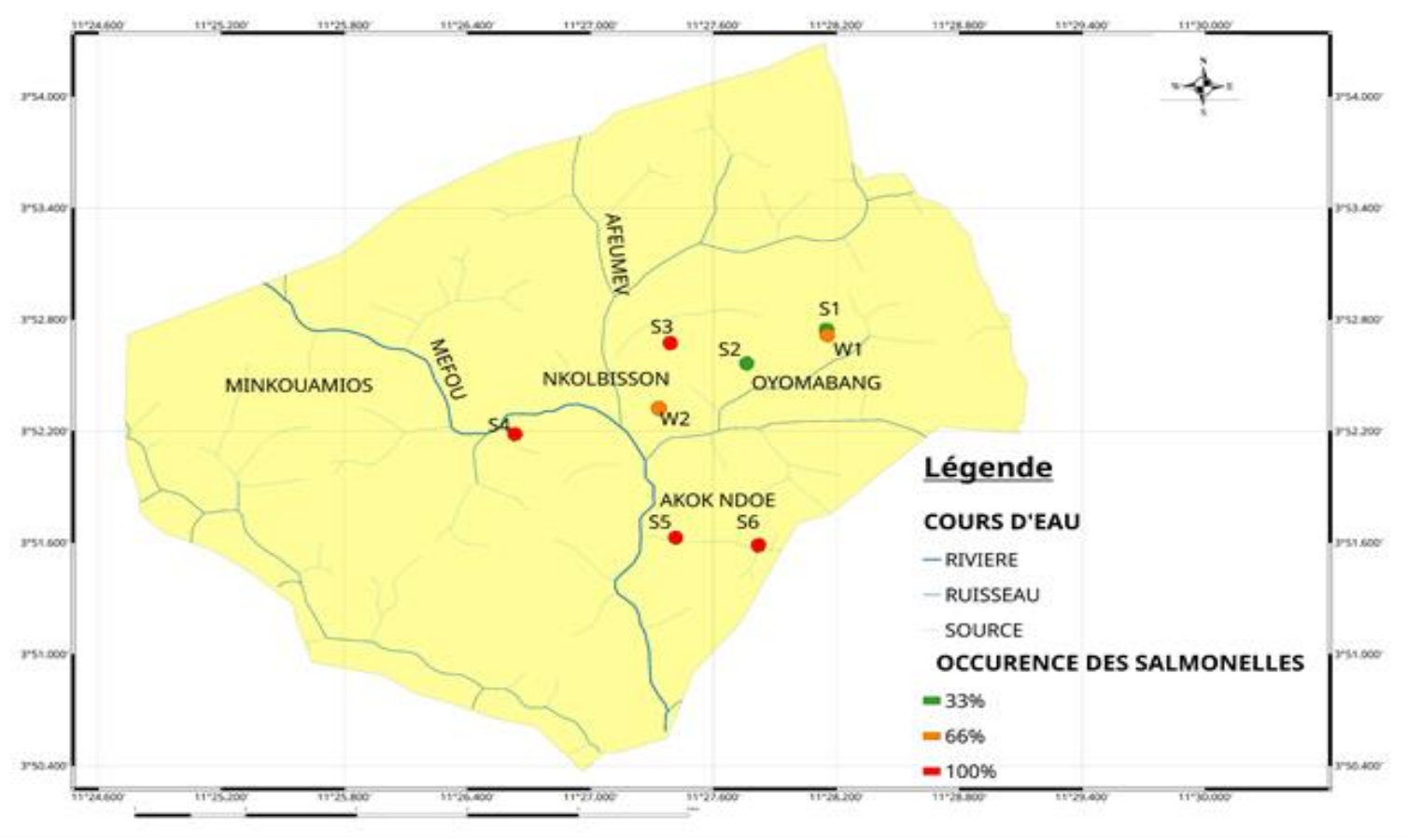

Figure 4 : Distribution des Salmonelles dans la zone d'étude.

Source: carte topographique Yaoundé 3d. NA-32XXIV à l'échelle 1/50 000 dessinée par IGN Paris, 1956, digitalisée par Diane MOUSSIMA, 2019. 


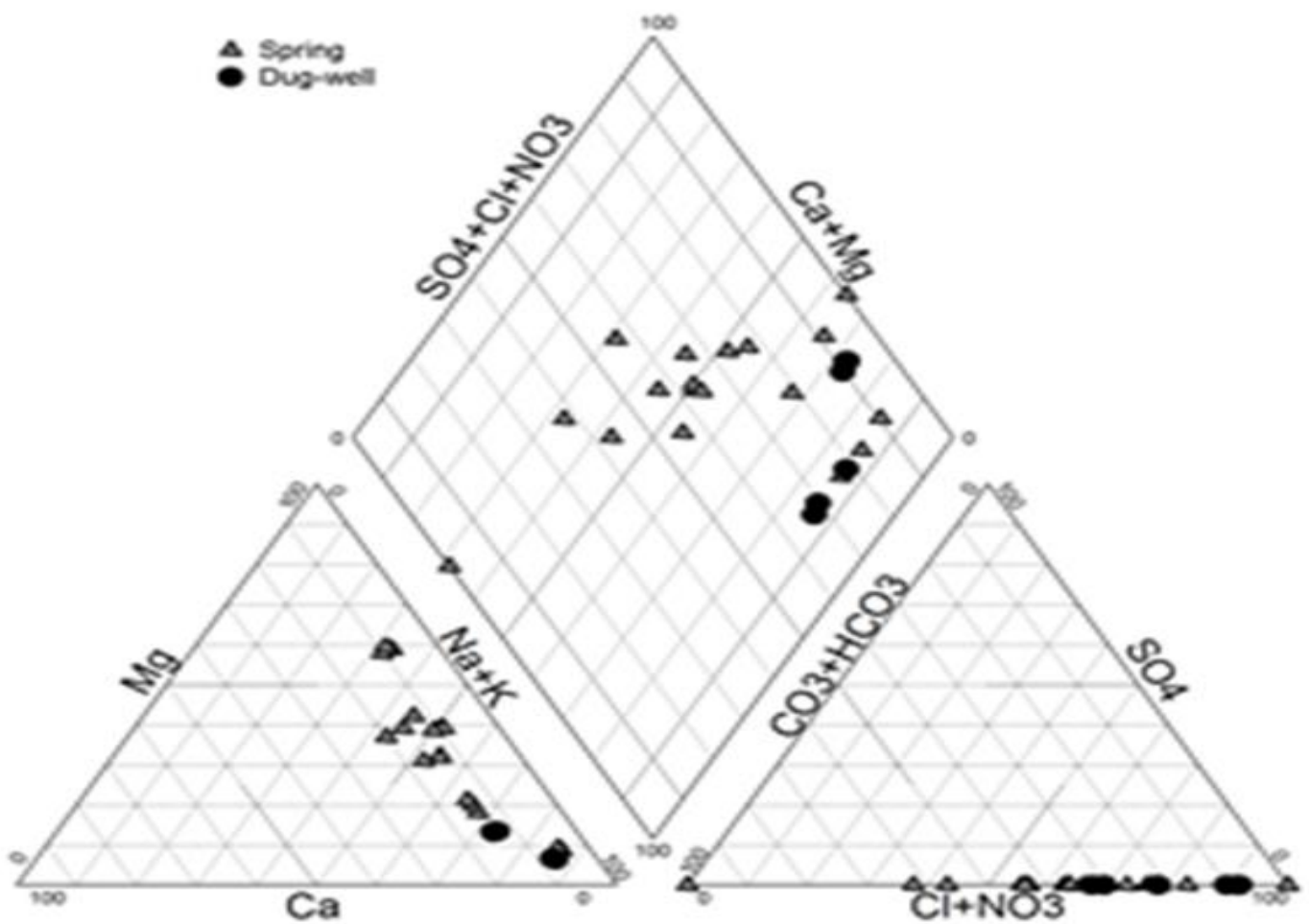

Figure 5: Diagramme de piper présentant le faciès chimique des eaux échantillonnées.

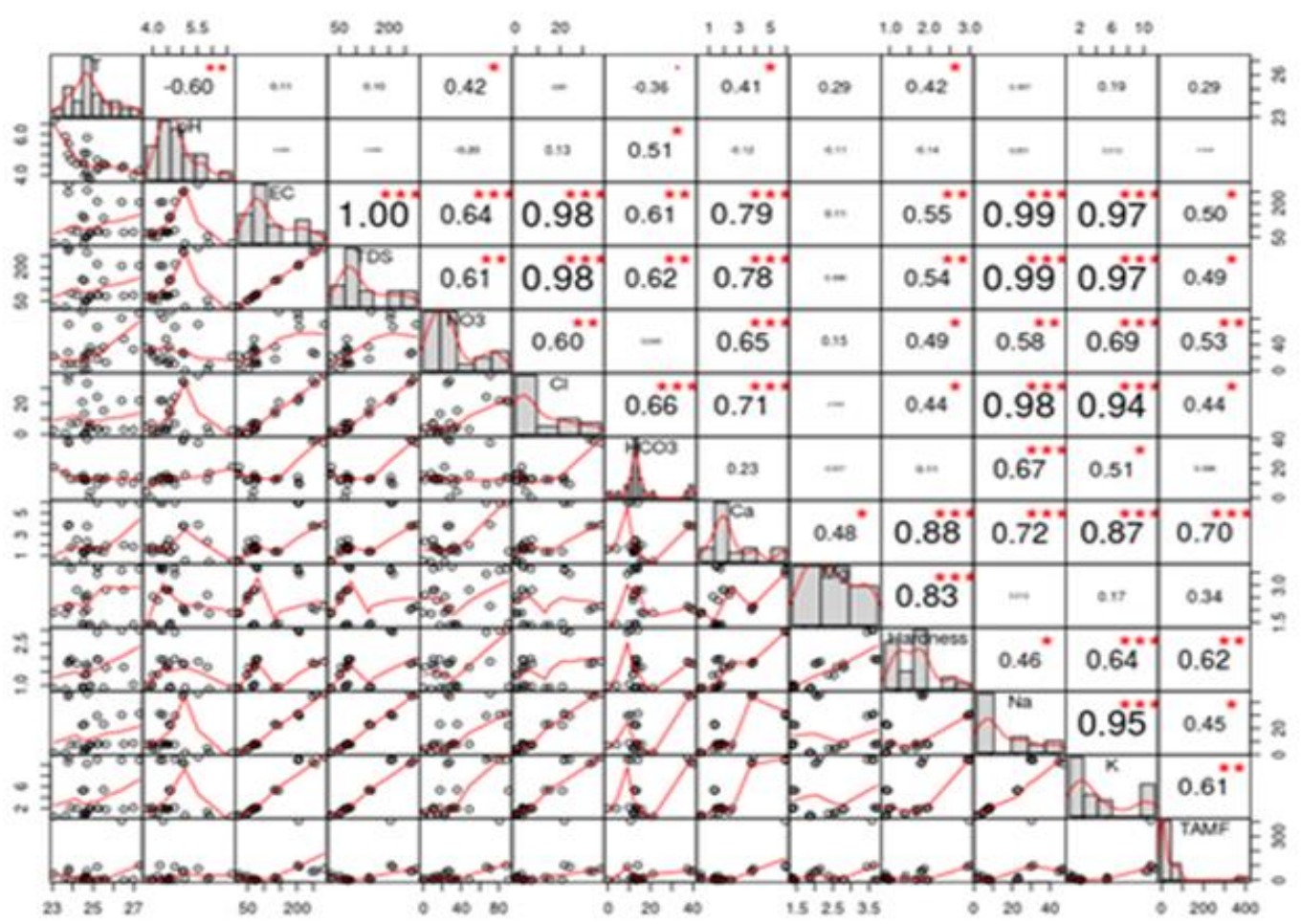

Figure 6: Matrice de corrélation de Spearman entre les paramètres physico-chimiques et entre les paramètres physico-chimiques et la FAMT. 
D. A. MOUSSIMA YAKA et al. / Int. J. Biol. Chem. Sci. 14(5): 1902-1920, 2020
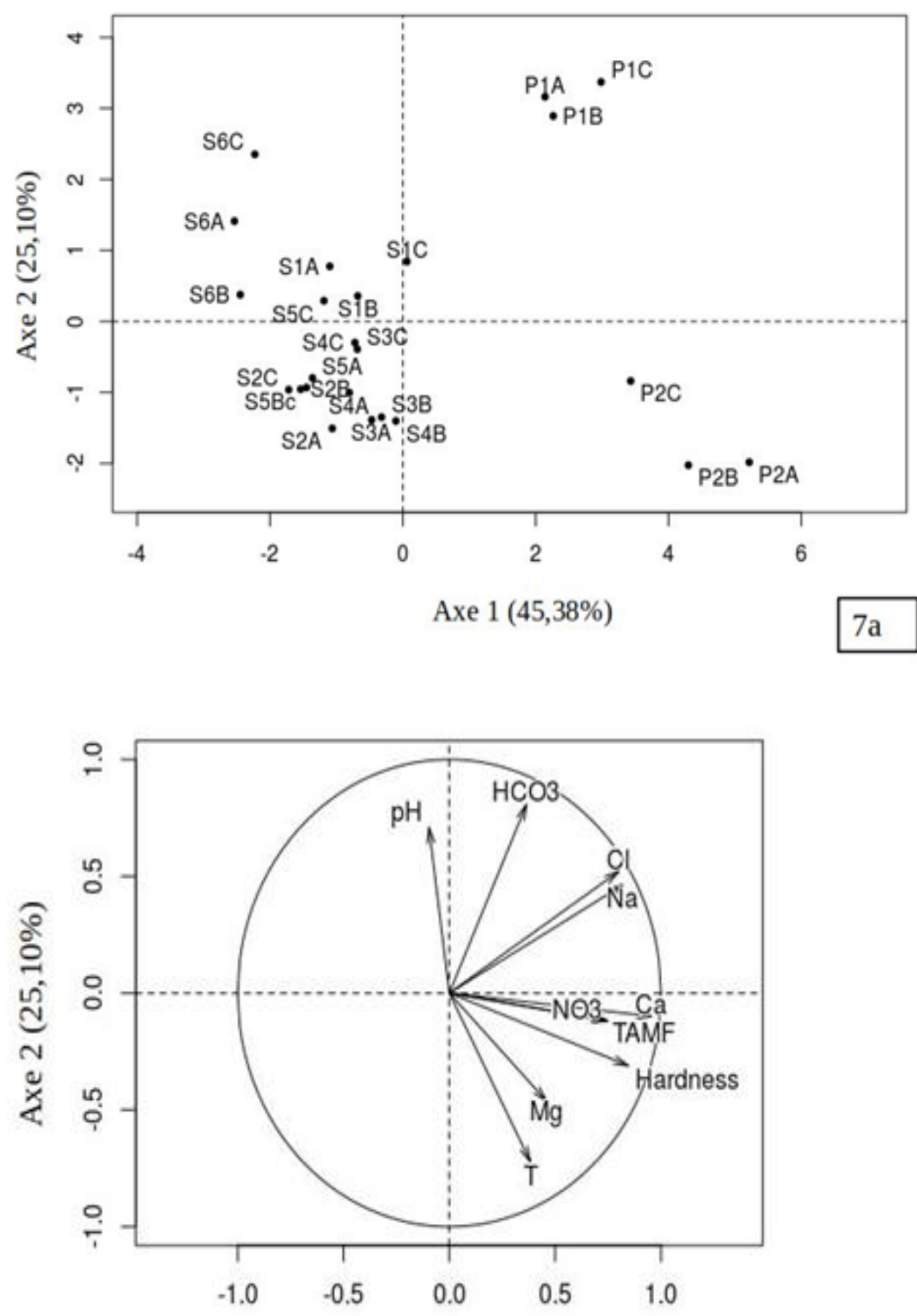

Axe $1(45,30 \%)$

Figure 7: Analyse en composantes principales des stations d'échantillonnage en fonction des variables analysées (7a: carte des stations ; 7b : carte des variables). 

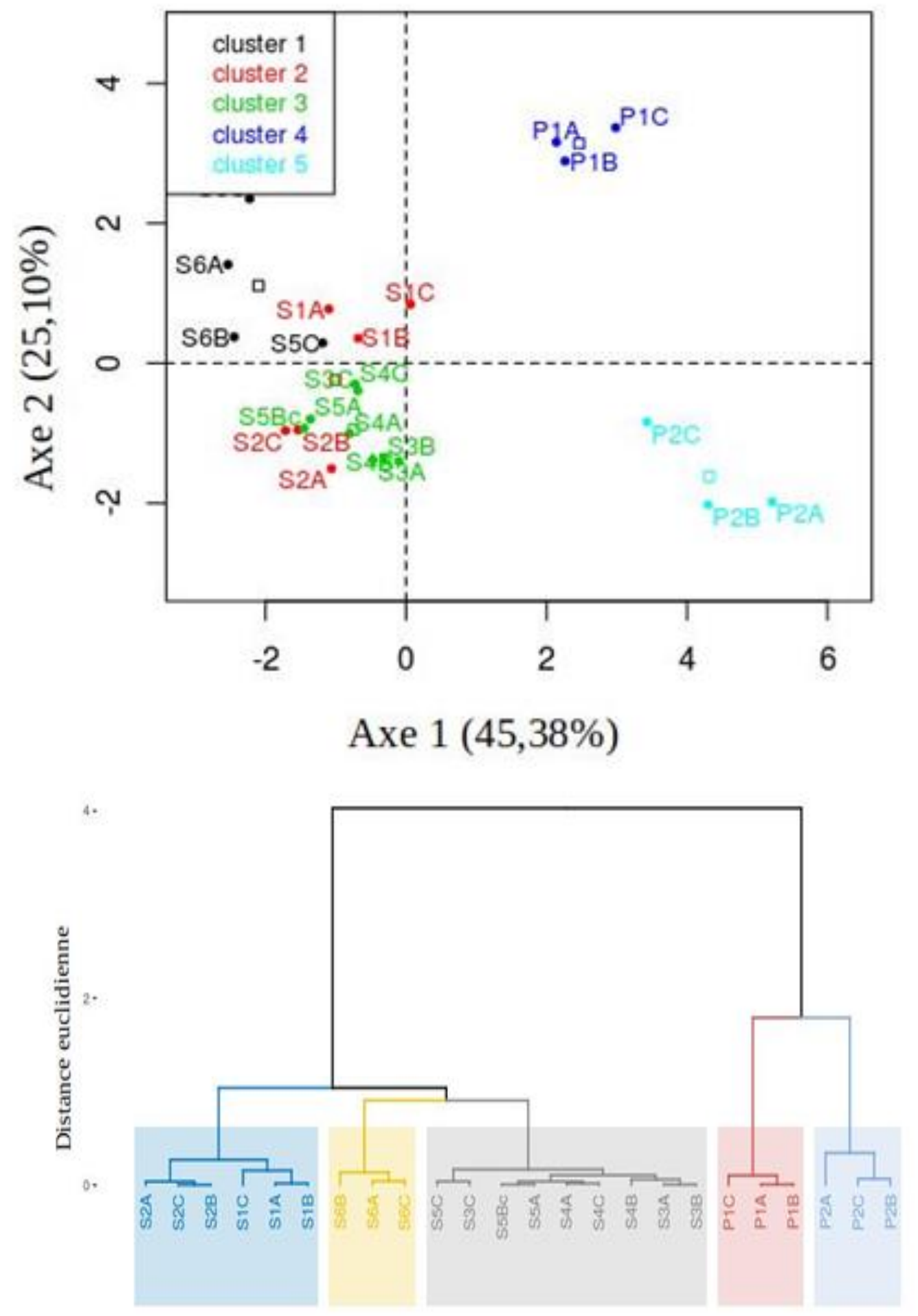

Figure 8: Classification ascendante hiérarchique des stations d'échantillonnage en fonction des variables analysées. 


\section{DISCUSSION}

Les valeurs moyennes de température des eaux échantillonnées (Tableau 3), restées sensiblement proches de celle de l'air témoigneraient du caractère libre de ces nappes. Celle-ci sont peu profondes et, subissent l'influence directe des eaux d'infiltration, leur principal moyen de recharge. Elles seraient donc plus exposées à la pollution.

Les valeurs médianes de $\mathrm{pH}$ (Tableau 3) indiquent que les eaux souterraines échantillonnées ont une tendance acide. $92 \%$ des prélèvements ont un $\mathrm{pH}$ inférieur à la valeur minimale fixée par la NC 207 (2014) qui est de 6,5. Ces résultats corroborent ceux obtenus conjointement par l'INS et le BGR (2013) $(90 \%$ de valeurs inférieures à 6,5) lors de leur étude sur la qualité des eaux de surface et souterraine dans la ville de Yaoundé. Une eau légèrement acide n'est pas dangereuse pour la santé des consommateurs. Cependant, un $\mathrm{pH}$ bas peut indiquer la présence d'un polluant dans l'eau ou encore, traduire une forte activité minéralisatrice des bactéries, à proximité de la nappe (Matini et al., 2009).

$\mathrm{La}$ très forte corrélation entre les solides totaux dissous et la conductivité électrique $(r=1)$ serait dû au fait que ces paramètres décrivent tous deux les sels inorganiques présents en solution. Selon Nono et al. (2009) et Yombi et al. (2013), les valeurs de conductivité électrique des eaux des nappes superficielles sur altérite en zone cristalline sont inférieures à $50 \mu \mathrm{S} / \mathrm{cm}$ et résultent principalement de la solubilisation des minéraux des roches. Les valeurs dépassant ces limites obtenues à toutes les stations excepté $\mathrm{S}_{6}$ indiquent un apport extérieur des ions. Ces derniers sont essentiellement les ions nitrates, chlorures, calcium, sodium, potassium et bicarbonates (Figure 6).

La roche-mère qui constitue le substratum géologique des sols de Yaoundé est une Embréchite à grenats, traversée selon les lieux, par de plus ou moins nombreux filons de quartz; dans ces roches, le magnésium est abondant, le potassium bien représenté et le sodium est faibles (Nzenti et Eno Belinga, 2001). La dominance des ions sodium et potassium dans ces eaux (Figure 5), conforte donc l'idée d'un apport autre que la roche mère en ces éléments. De même la dominance des anions chlorures et nitrates (Figure 5) indiquent clairement une action anthropique. En effet, des teneurs en nitrates supérieures à $10 \quad \mathrm{mg} / \mathrm{l}$ reflètent une contamination anthropique selon Ollagnier et al. (2007) et Kloppmann (2003). Les points $\mathrm{S}_{1}, \mathrm{~W}_{1}$ et $\mathrm{W}_{2}$ ont présenté des concentrations en nitrate $>50 \mathrm{mg} / \mathrm{l}$, valeur limite acceptable de la NC 207 (2014). Ces concentrations trop élevées sont dangereuses pour la santé des consommateurs, car peuvent causer la méthémoglobinémie chez les enfants, des cancers chez l'adulte par formation des nitrosamines (Jalili et al., 2018). Cette forte pollution azotée a également été relevée par Yombi et al. (2013) dans les eaux souterraines du bassin versant de la Mingoa, par Ntep et al. (2014) dans les eaux souterraines du bassin versant de la Biyémé à Yaoundé, ainsi que par Nzeket et al. (2019) dans les eaux souterraines de la ville d'Edea. La forte activité maraîchère dans la zone étudiée pourrait justifier ce résultat. La proximité des maisons d'habitation permettrait également d'attribuer cette pollution azotée des eaux à la décomposition de la matière fécale, et des ordures ménagères, laissant croire un risque de contamination bactérienne. La corrélation significative entre le nitrate et la FAMT $(r=0.53)$ conforte cette hypothèse.

La présence d'entérocoques ou de streptocoques dans les eaux analysées (Figures 2 et 3 ) témoigne d'une contamination fécale de celles-ci. Elle pourrait être due à une diffusion par infiltration ou par diffusion latérale (Chippaux et al., 2002 ; Nola et al., 2006) à travers les porosités du sol des eaux souillées, leur ruissellement (car ces puits ne respectent pas les normes de construction), ou encore à une contamination directe de la nappe d'eau par des objets souillés. Les entérocoques intestinaux et les streptocoques fécaux font partie de la flore intestinale normale. Cependant, en raison de la sousalimentation et de la sur-infestation de la population d'un pays en voie de développement comme le Cameroun, ils pourraient causer des dommages à 
l'organisme pouvant conduire à la mort. Leur pouvoir pathogène est lié aux maladies telles que des infections urinaires, des infections digestives et même des endocardites (Isnard, 2017).

Salmonella $s p$ isolé dans les eaux analysées est une bactérie d'un très grand intérêt sanitaire du fait de sa virulence et, de sa résistance à nombreux traitements (Kashosi et al., 2018). Sa présence dans tous les points d'échantillonnage (Figure 4) reflète la gravité de l'état de pollution de ces eaux. La forme typhique de cette bactérie est responsable chez l'homme, de la fièvre typhoïde. Cette pathologie peut se compliquer en péritonite, cholécystite, myocardite et ostéite avec une forte mortalité et morbidité dans les pays en développement (Aubry et Gaüzère, 2020). Un risque sanitaire inquiétant lié à l'état de pollution microbiologique avancée des eaux souterraines du bassin versant de la Mingoa (Yaoundé) a également été révélé par Yombi et al. (2013). Nzouebet et al. (2019) ont par ailleurs trouvés, lors de leur étude sur l'évaluation des pratiques de gestion des boues de vidange dans la ville de Yaoundé, que la fièvre typhoïde est la maladie entérique la plus répandue dans les ménages. Cette forte prévalence de la fièvre typhoïde a également été observée dans d'autres régions du Cameroun notamment celle du Nord-Ouest (Njoyim et al., 2019).

L'ACP (Figure 7) ainsi que le dendrogramme (Figure 8) réalisés à partir des résultats obtenus permettent de classer les points d'échantillonnage en groupes, selon l'ordre croissant de pollution suivant : $\left\{\mathrm{S}_{6}\right\}$, $\left(\mathrm{S}_{3}, \mathrm{~S}_{4}, \mathrm{~S}_{5}\right),\left(\mathrm{S}_{1}, \mathrm{~S}_{2}\right),\left\{\mathrm{W}_{1}\right\},\left\{\mathrm{W}_{2}\right\}$. Cependant, du point de vue pathogénique, le singleton $\left\{S_{6}\right\}$ et le groupe $\left(S_{3}, S_{4}, S_{5}\right)$, bien qu'étant à priori les moins pollués, seraient les plus à craindre (Figure 4). La conformité d'une eau vis-à-vis de certains paramètres physicochimiques et bactériologique (FAMT), ne garantit pas sa potabilité.

\section{Conclusion}

Les données collectées au cours de cette étude ont permis de dresser un portrait de la qualité physico-chimique et bactériologique des eaux souterraines des quartiers OyomAbang, Nkolbisson et AkokNdoé. Ces eaux présentent des signes de contamination anthropique. Dans les stations $\mathrm{S}_{1}, \mathrm{~W}_{1}$ et $\mathrm{W}_{2}$ les teneurs en nitrates sont supérieures à la norme. La qualité microbiologique de toutes les eaux échantillonnées est inacceptable. Les indicateurs de pollution fécale ainsi que des bactéries du genre Salmonelle y ont été isolés à toutes les campagnes d'échantillonnage. Au vu de la forte sollicitation de ces ressources dans de multiples besoins ménagers tels que, la lessive, la vaisselle, la cuisine, l'hygiène corporelle et même la boisson, il apparait un risque de développement par la population de nombreuses maladies d'ordre entéropathologique et métabolique telles que la méthémoglobinémie, et des cancers. Comme solution, la population devrait adopter des attitudes visant à limiter la contamination de la nappe phréatique (assainir leur environnement, respecter les règles d'hygiène et les normes de construction des puits et sources). Elle devrait traiter les eaux souterraines avant toute utilisation. Les pouvoirs publics devraient mettre en place des programmes de surveillance de la qualité des eaux souterraines, ainsi que des programmes d'information et d'éducation des populations afin de leur inculquer des comportements favorables à la gestion de ces points d'eau.

\section{CONFLIT D'INTÉRÊTS}

Les auteurs déclarent qu'il n'y a pas de conflit d'intérêts lié à la publication cet article.

\section{CONTRIBUTIONS DES AUTEURS}

MYDA et TAA ont effectués la collecte des eaux sur le terrain. Avec FTBH ils ont effectués les analyses microbiologiques. MYDA, TAA et AA ont effectués l'étude bibliographique. ZZB et NAB ont aidés dans l'analyse physicochimique des eaux. JNLLT a effectué les analyses statistiques des données. MMYC a fourni certaines normes et réactifs. MYDA a écrit le manuscrit. Tous les auteurs ont lu, amélioré et approuvé la version finale du document. 


\section{REMERCIEMENTS}

Nos remerciements vont à l'endroit de l'Institut de Recherche Agricole pour le Développement pour son aide dans la réalisation des analyses physico-chimique et bactériologiques à travers le Laboratoire accrédité d'Analyses des Sols, Plantes, Eaux et Engrais (LASPEE) et le Laboratoire de Phytopathologie. Nous remercions également l'Université de Douala, précisément le Professeur Annie NGONO NGANE chef du Département de Biochimie, pour son soutien technique. Des remerciements sincères également à M. AGOUME Victor pour son encadrement dans la rédaction du présent manuscrit.

\section{RÉFÉRENCES}

Aubry P, Gaüzère B. 2019. Les salmonelles, Médecine Tropicale. Centre René Labusquière, Institut de Médecine Tropicale, Université de Bordeaux, p.9

Aulagnier S, Vittecoq B. 2007. Suivi de la qualité des eaux souterraines de Martinique, campagne de saison des pluies 2006: résultats et interprétation. Rapport Bureau de recherches géologiques et minières BRGM/RP55499-FR, p. 77.

Banque Mondiale-Ministère de l'Habitat et du Développement Urbain. 2017. Cadre de gestion environnementale et sociale (CGES). Rapport final du projet, p. 129, [en ligne] http://documents.worldbank.org/curated/ en/692541493629902004/pdf/SFG3295REPLACEMENT-EA-P156210PUBLIC.pdf. Consulté le 14/10/20

Chippaux JP, Houssier S, Gross P, Bouvier C, Brissaud F. 2002. Étude de la pollution de l'eau souterraine de la ville de Niamey. Niger. Bull. Soc. Pathol. Exot., 94 (2) : 119-123

DSCE (Document de Stratégie pour la croissance et l'emploi). 2009. Cameroun, p. 174.

INS-BGR (Institut National de la StatistiqueInstitut Fédérale des Géosciences et des Ressources Naturelles). 2013. Étude pilote sur la pollution des eaux de surface et souterraines à Yaoundé et impact sur la santé des populations riveraines (EPESS). Rapport technique du projet BMZ PN 2002.3510.1 BGR 05-2203-54, p. 194. Yaoundé, Hanovre, Bonn.

Isnard C. 2017. Enterococcus spp. : entre pathogènes opportunistes et probiotiques. Thèse de $\mathrm{Ph}$. D, Université de Caen Normandie, p. 299.

Jalili D, RadFard M, Soleimani H, Nabavi S, Akbari H, Akbari H. 2018. Data on nitrate-nitrite pollution in the groundwater resources a Sonqor plain in Iran. Data in Brief, 20: 394-401. DOI: https://doi.org/10.1016/j.dib.2018.08.023

Kashosi MT, Muhandule BA, Mwenebitu LD, Mihuhi N, Mutendela KJ, Mubagwa K. 2018. Antibio-résistance des souches de Salmonella spp isolées d'hémocultures à Bukavu en RD Congo. Pan African Medical Journal, 29(42): 1-8. DOI: 10.11604/pamj.2018.29.42.13456

Klopp Mann W. 2003. Étude isotopique de la pollution azotée de la nappe d'Alsace entre Sierentz et Ottmarsheim. Rapport Bureau de recherches géologiques et minières BRGM/RP-52331 -FR, p. 69.

Matini L, Mouton JM, Kongo-Mantono MS. 2009. Evaluation hydro-chimique des eaux souterraines en milieu urbain au Sud-Ouest de Brazzaville, Congo. Afrique Science, 05 (1) : 82-98.

NC (Norme Camerounaise) : 2014, Norme pour l'eau destinée à la consommation humaine. 2014. ANOR.

NF EN ISO (Norme Française, European Norm, International Organization for Standardization) 6222, Qualité de l'eau Dénombrement des micro-organismes revivifiables - Comptage des colonies par ensemencement dans un milieu de culture nutritif gélosé. 1999. Edn. AFNOR, p. 4.

NF EN ISO (Norma Française, European Norm, International Organization for Standardization) 7899-2, Qualité de l'eau- Recherche et dénombrement des entérocoques intestinaux-Partie 2 : Méthode par filtration sur membrane. 2000. Ed. Edn. AFNOR, p. 8.

NF ISO (Norma Française, European Norm, International Organization for 
Standardization) 19250, Qualité de l'eauRecherche de Salmonella spp. 2010. Edn. AFNOR, p. 24.

Njoyim IK, Kengni L, Tamen J, Awah MT, Tamungang BEN. 2019. Spring quality assessment and effects on the health of inhabitants of Santa Sub-Division, North West Region, Cameroon. Int. J. Biol. Chem. Sci., 13(6): 2894-2913. DOI: https://dx.doi.org/10.4314/ijbcs.v13i6.37

Nola M, Njiné T, Kemka N, Zébazé TSH, Servais P, Messouli M, Boutin CI, Monkiedje A, Foto MS. 2006. Transfert des bactéries fécales vers une nappe phréatique à travers une colonne de sol en région équatoriale: influence de la charge en eau appliquée en surface. Revue des Sciences de l'Eau, 19(2): 69149. DOI : https://doi.org/10.7202/013044.

Nono A, Likeng JDH, Wabo H, Youmbi TJG, Biaya S. 2009. Influence de la nature lithologique et des structures géologiques sur la qualité et la dynamique des eaux souterraines dans les hauts plateaux de l'Ouest-Cameroun. Int. J. Biol. Chem. Sci., 3(2): 218-239.

Ntep F, Kengne IM, Mboudou EG, Nyochembeng N, Ekodeck GE. 2014. Influence of seasonal dynamics on groundwater resources quality in semitropical urban zone: case of the Biyémé upper stream catchment (Yaounde, Cameroon). Int. J. Biol. Chem. Sci., 8(3): 1319-1335.

DOI: http://dx.doi.org/10.4314/ijbcs.v8i3.44

Nzeket AB, Moyo KB, Aboubakar A, Youdom YAS, Moussima YDA, Zing ZB, Sulem YNN, Mama AC, Mfopou MYC. 2019. Assessment of Physicochemical and Heavy Metal Properties of Groundwater in Edéa (Cameroon). American Journal of Water Resources, 7(1): 1-10. DOI: 10.12691/ajwr-7-1-1.
Nzenti JP, Belinga ESM. 2001. Géologie de la région de Yaoundé et ses environs in excursions géologiques dans le sud du Cameroun. 12ème Conférence Internationale de la Société Géologique Africaine, Université de Yaoundé I; 1930

Nzouebet LAW, Kengne SE, Wafo DVG, Wanda C, Rechenburg A, Noumsi KMI. 2019. Assessment of the faecal sludge management practices in households of a sub-Saharan Africa urban area and the health risks associated: the case study of Yaoundé, Cameroon. Int. J. Biol. Chem. Sci., 13(5): 1-23. DOI : https://dx.doi.org/10.4314/ijbcs.v13i5.1S

OMS. 2004. Directives de qualité pour l'eau de boisson, volume 1 , recommendations (3ème edn). OMS, Genève, Suisse, p. 110.

Rapport national de contribution du Cameroun. 2015. Troisième conférence des nations unies sur le logement et le développement durable en milieu urbain (Habitat III), p. 47, [en ligne] www.habitat3.org/wpcontent/uploads/Cameroon-Rapportnational-Habitat-III-Version-fev-16.pdf. Consulté le 08/10/2019.

Rodier J, Lègue B, Merlet N, Brunet R. 2009. L'analyse de l'eau (9ème edn). Ed. Dunod : Paris ; 1526.

UNRIC. 2020. Objectifs de développement durable (ODD), sélection de ressources en ligne. Fiche d'information, [en ligne] https://unric.org/fr/wpcontent/uploads/sites/2/2020/02/sdgsfrench.pdf. Consulté le 05/03/2020.

Youmbi TGJ, Feumba R, Njitat TV, De Marsily G, Ekodeck GM. 2013. Pollution de l'eau souterraine et risques sanitaires à Yaoundé au Cameroun. Comptes Rendus Biologies, 336: $\quad 310-$ $316 . \quad$ DOI : https://doi.org/10.1016/j.crvi.2013.04.01 3. 